\title{
Case Report: Bilateral Pneumothoraces Due To Targeted Tumor Therapy With Regorafenib in a Young Woman With Metastatic Colorectal Cancer
}

Tobias Rachow ( $\sim$ tobias.rachow@med.uni-jena.de)

Universitätsklinikum Jena: Universitatsklinikum Jena

Tim Sandhaus

Universitätsklinikum Jena: Universitatsklinikum Jena

Thomas Ernst

Universitätsklinikum Jena: Universitatsklinikum Jena

Helmut Schiffl

LMU München: Ludwig-Maximilians-Universitat Munchen

Susanne M. Lang

Universitätsklinikum Jena: Universitatsklinikum Jena

\section{Case report}

Keywords: regorafenib, pneumothorax, pulmonary metastases, colorectal cancer

Posted Date: October 26th, 2021

DOl: https://doi.org/10.21203/rs.3.rs-991839/v1

License: (9) This work is licensed under a Creative Commons Attribution 4.0 International License. Read Full License 


\section{Abstract \\ Background:}

Colorectal cancer is one of the most common cancer types, frequently metastasizing into the lungs. Treatment options have been vastly improved over the last years. With the increasing use of targeted therapies novel and rare adverse effects can be seen. In this report, we present the case of recurrent spontaneous bilateral pneumothorax due to fulminant tumor necrosis during the administration of regorafenib in a heavily pretreated patient with multiple lung metastases from colorectal cancer.

\section{Case presentation:}

A 43-year-old woman presented in our oncology department with chest pain and dyspnea. The patient was diagnosed with colorectal cancer seven years earlier and had received chemoradiation, surgery and multiple chemotherapies, before she was started on regorafenib because of progressive pulmonary metastases. Clinical examination revealed no breath sounds in the right hemithorax. The patient was tachycardic and orthopneic. Computed tomography scans demonstrated cavitation of former nodular bilateral pulmonary metastases. After drainage and resolution of the right-sided pneumothorax the patient returned eleven days later with recurrent dyspnea, chest pain and subcutaneous emphysema. Bilateral pneumothoraces were treated with chest tubes. Due to left-sided tension pneumothorax videoassisted thoracoscopy and bilateral pleurodeses were performed. Persistent air leaks with severe pain and pulmonary infiltrates led to the death of the patient.

\section{Conclusions:}

Our case illustrates the effectiveness of regorafenib in a highly pretreated patient. However, in our patient the ensuing cavitation of the multiple nodes led to recurrent pneumothoraces and associated infectious complications. Therefore, special surveillance should be implemented to detect potential transformation of solid pulmonary metastases during treatment with this multi-kinase inhibitor.

\section{Background}

Metastatic colorectal cancer (mCRC) is the third most common cause of cancer mortality worldwide. Up to $50 \%$ of patients present with or develop metastatic disease mainly in the liver and the lungs $[1,2]$. The management of $\mathrm{mCRC}$ has undergone a strategic revolution in imaging, innovations in surgical and local ablative techniques and unprecedented advances in systemic medical therapy (cytostatic agents, immunotherapies and targeted therapies). Molecular profiling of $\mathrm{mCRC}$ unfolded biological determinants of the disease and led to better treatment selection for more efficient therapeutic strategies. Moreover, it reduced the need for cytotoxic therapies with its associated high level of toxicities. However, the use of targeted therapies may be associated with novel and rare adverse effects. 
In this report, we present the rare case of recurrent spontaneous bilateral pneumothorax due to rapid tumor reduction during the administration of oral the multi-kinase inhibitor regorafenib in a patient with multiple lung metastases from mCRC.

\section{Case Presentation}

In October 2020 a 43-year-old woman presented in our oncology department with chest pain and dyspnea. Clinical examination revealed no breath sounds in the right hemithorax. The patient was tachycardic and orthopneic. Chest x-ray showed right-sided pneumothorax (figure 1). Computed tomography (CT) scans revealed cavitation of former nodular bilateral pulmonary metastases (figure 2) but no progressive disease. Pneumothorax resolved after insertion of a chest tube and suction therapy. Dyspnea resolved promptly and the patient could be discharged a few days later after removal of the chest tube.

Eleven days later, the patient was readmitted due to recurrent dyspnea, chest pain and subcutaneous emphysema. Chest x-ray not only showed recurrence of the right-sided pneumothorax but also another pneumothorax in the left apex of the lung. After insertion of bilateral chest tubes subsequent x-rays showed complete resolution of the left-sided and partial resolution of the right-sided pneumothorax. Four days later, the patient reported once more massive chest pain. Chest $x$-ray revealed left-sided tension pneumothorax and another chest tube was inserted. Furthermore, video-assisted thoracoscopy and bilateral pleurodeses were performed. However, the chest tubes could not be removed due to persistent air leaks. In addition, antibiotic treatment was started because of pneumonia. After the surgical procedures, chest pain worsened continuously, and the patient received specialized pain treatment and palliative sedation. Finally, the patient died 30 days after readmission due to infectious complications and persistent air leaks.

\section{Medical history}

The patient was diagnosed with colorectal cancer in August 2013. The initial clinical staging was cT3 cN1 cM0 (UICC IIIB). After neoadjuvant chemoradiation, lower anterior resection was performed in November 2013. Resection was complete (R0), final pathological staging showed a stage IIIC (ypT3 pN2b $(8 / 13)$ cM0). Hereditary tumor syndromes were excluded by genetic analysis. Adjuvant monochemotherapy with 5 -fluorouracil (5-FU) was started but had to be modified to an oral therapy with capecitabine because of poor tolerance. The patient received three complete cycles. Follow-up examinations showed no signs for local tumor recurrence or metastases. In November 2014 two pulmonary metastases were detected, which could be resected. From February to April 2015 another chemotherapy (FUFOX-scheme $=$ fluorouracil/folinic acid /oxaliplatin) was applied. Tolerance was poor with treatment associated anaphylactic reactions, most likely caused by oxaliplatin, infection and chest pain. Consequently, chemotherapy had to be discontinued. In November 2015, a solitary left-sided pulmonary metastasis was diagnosed and resected. Molecular testing showed a NRAS mutation and chemotherapy with 5-FU, folic acid, irinotecan and bevacizumab was initiated. Due to severe side effects 
the therapy was switched to mitomycin and bevacizumab. In September 2016 relapse was detected with pulmonary metastases and bilateral pleural effusions. The patient was switched to 5-FU, folic acid and bevacizumab which resulted in stable disease with stable to slow growing pulmonary metastases until October 2018. In November 2018 wedge-resection of a pulmonary metastasis was performed for whole genome sequencing, which showed a pathogenic tumor protein P53 (TP53)-mutation, a NRAS G12D mutation and a high mutational burden. In January 2019 severe diarrhea developed and the dose of 5-FU had to be reduced. Follow-up CT-scans showed slowly progressing pulmonary metastases. In June 2019 bevacizumab was replaced by aflibercept while continuing a dose reduced regimen of 5-FU, folic acid, and irinotecan. In January 2020, a CT-scan suggested local recurrence of the tumor accompanied by perirectal abscesses. Finally, surgical resection showed no malignancy but local infection. Chemotherapy was continued until June 2020 when imaging showed disease progression. Intravenous therapy was discontinued and switched to an oral therapy with trifluridine/tipiracil. In September two brain metastases were detected in a routine magnetic resonance imaging (MRI) scan and a therapy with regorafenib was initiated before immunotherapy could be instituted as off-label use. Over the time, the patient had received a cumulative dose of $171,200 \mathrm{mg}$ fluorouracil.

\section{Discussion And Conclusions}

Regorafenib is a multikinase inhibitor. Antiangiogenic activity is mainly mediated by binding to vascular endothelial growth factor receptor (VEGFR) 1-3. Moreover, it inhibits tyrosine-protein kinase KIT- and rearranged during transfection (RET)-signaling cascades which are important for tumor development and progression. Activation of fibroblast growth factor receptor (FGFR) and platelet derived growth factor receptor (PDGFR)-kinases, which are inhibited by the substance as well, has been implicated in tumor progression and metastasis. Regorafenib showed survival benefits in MCRC which had progressed after standard therapies [3]. The substance was approved for the treatment of mCRC by the Food and Drug Administration in 2012 and in 2013 by the European Medical Association (EMA). A recently published trial confirmed the effects in a real-world setting [4].

Our heavily pretreated patient responded surprisingly well to regorafenib with complete transformation of the pulmonary metastases to cavities. This effect has been recently described in a few case reports $[5,6]$. The exact mechanism of cavity formation remains unclear but central tumor necrosis caused by antiangiogenic activity is suspected to be the most likely cause [7, 8].

In our case relapsing pneumothoraces could not be sufficiently controlled by chest drainage and surgery was necessary. We terminated the treatment after the initial pneumothorax. However, further cavitation and pneumothoraces occurred even after discontinuation of treatment.

Regorafinib is an effective agent for the treatment of colorectal cancer in pretreated patients. Large randomized, double-blind, multinational, phase 3 clinical trials showed benefits regarding progressionfree and overall survival $[3,9]$. Our case illustrates the effectiveness of regorafenib in a highly pretreated patient. However, in our patient the ensuing cavitation of the multiple nodes led to recurrent 
pneumothoraces and associated infectious complications. Therefore, special surveillance should be implemented to detect potential transformation of solid pulmonary metastases during treatment with this multi-kinase inhibitor.

\section{List Of Abbreviations}

$\mathrm{mCRC}=$ metastatic colorectal cancer

$\mathrm{CT}=$ computed tomography

5 -FU = 5-fluorouracil

FUFOX = fluorouracil/folinic acid /oxaliplatin

KRAS = oncogen detected in Kirsten RAt Sarcoma virus

TP53 = tumor protein p53

$\mathrm{MRI}=$ magnetic resonance imaging

VEGFR = vascular endothelial growth factor receptor

$\mathrm{KIT}$ = tyrosine-protein kinase $\mathrm{KIT}$

RET = rearranged during transfection

FGFR $=$ fibroblast growth factor receptor

PDGFR = platelet derived growth factor receptor

EMA = European Medical Association

\section{Declarations}

Ethics approval and consent to participate - not applicable (case report)

Consent for publication - the patient's husband consented for publication of this case report

Competing interests - all authors declare that there is no conflict of interest

Funding - there was no funding

Authors' contributions: TR, TS, TE, HS and SML contributed to the writing of the manuscript, all authors have read and approved the manuscript 
Acknowledgements: The authors are grateful to the patient's family for their permission to publish this case report.

All relevant data are included in this published article.

\section{References}

1. Biller LH, Schrag D. Diagnosis and Treatment of Metastatic Colorectal Cancer: A Review. Jama. 2021;325(7):669-85.

2. Väyrynen V, Wirta EV, Seppälä T, Sihvo E, Mecklin JP, Vasala K, et al. Incidence and management of patients with colorectal cancer and synchronous and metachronous colorectal metastases: a population-based study. BJS open. 2020;4(4):685-92.

3. Grothey A, Van Cutsem E, Sobrero A, Siena S, Falcone A, Ychou M, et al. Regorafenib monotherapy for previously treated metastatic colorectal cancer (CORRECT): an international, multicentre, randomised, placebo-controlled, phase 3 trial. Lancet. 2013;381(9863):303-12.

4. Ducreux M, Petersen LN, Öhler L, Bergamo F, Metges JP, de Groot JW, et al. Safety and effectiveness of regorafenib in patients with metastatic colorectal cancer in routine clinical practice in the prospective, observational CORRELATE study. European journal of cancer (Oxford, England: 1990). 2019;123:146-54.

5. Kawasaki K, Hamamoto Y, Adachi M, Kanai T, Takaishi H. Early tumor cavitation with regorafenib in metastatic colorectal cancer: A case report. Oncology letters. 2016;11(1):231-3.

6. De Stefano A, De Luca G, Romano C. From Cavitation of Lung Metastases to Pneumothorax: An Amazing Response to Regorafenib in Metastatic Colorectal Cancer? A Case Report. Archives of Clinical and Medical Case Reports. 2020;04(01).

7. Marom EM, Martinez CH, Truong MT, Lei X, Sabloff BS, Munden RF, et al. Tumor cavitation during therapy with antiangiogenesis agents in patients with lung cancer. J Thorac Oncol. 2008;3(4):351-7.

8. Crabb SJ, Patsios D, Sauerbrei E, Ellis PM, Arnold A, Goss G, et al. Tumor cavitation: impact on objective response evaluation in trials of angiogenesis inhibitors in non-small-cell lung cancer. $\mathrm{J}$ Clin Oncol. 2009;27(3):404-10.

9. Li J, Qin S, Xu R, Yau TC, Ma B, Pan H, et al. Regorafenib plus best supportive care versus placebo plus best supportive care in Asian patients with previously treated metastatic colorectal cancer (CONCUR): a randomised, double-blind, placebo-controlled, phase 3 trial. Lancet Oncol. 2015;16(6):619-29.

\section{Figures}




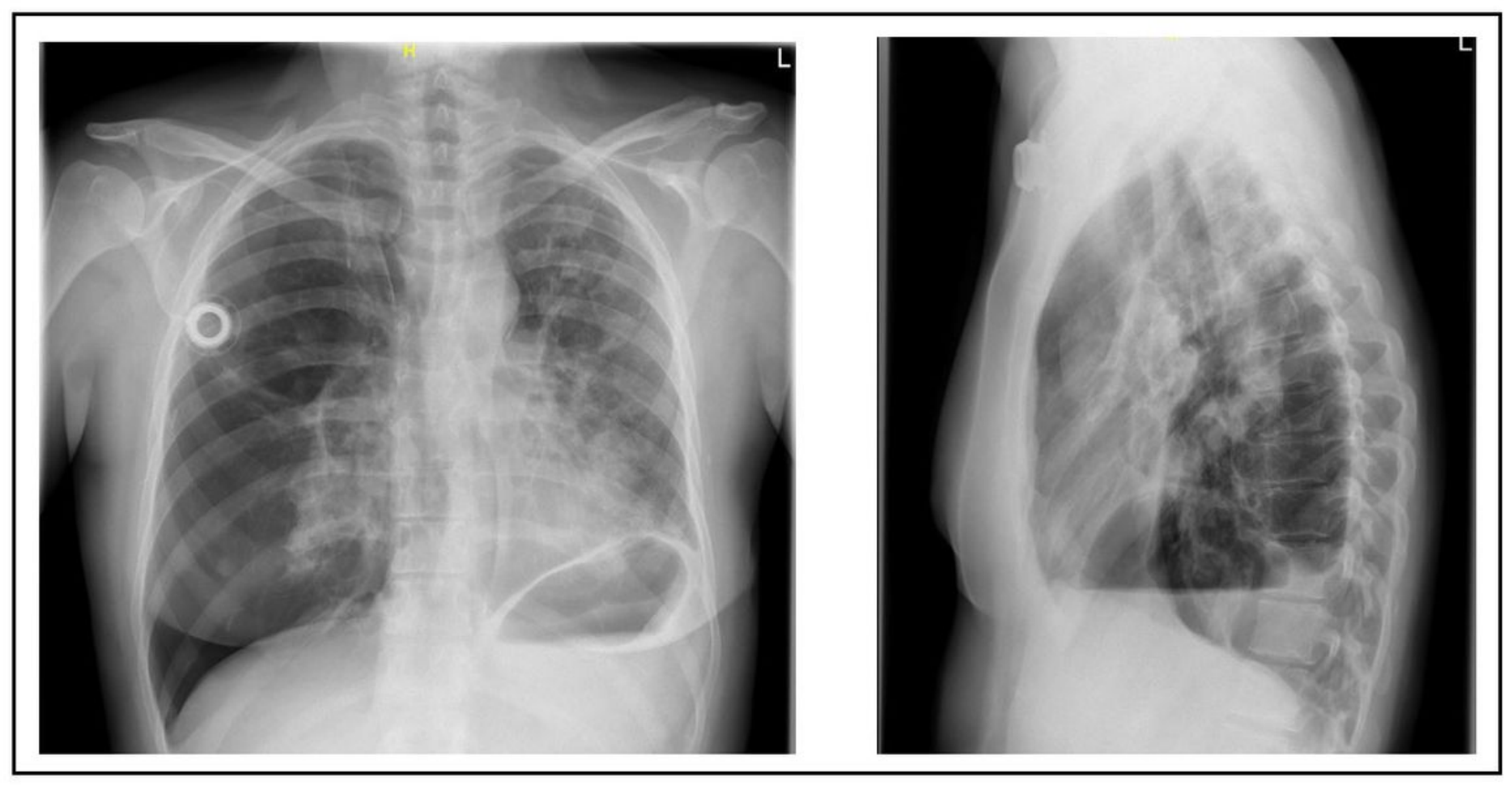

Figure 1

chest x-ray for the reason of suddenly developed chest pain and shortness of breath showing right sided pneumothorax 

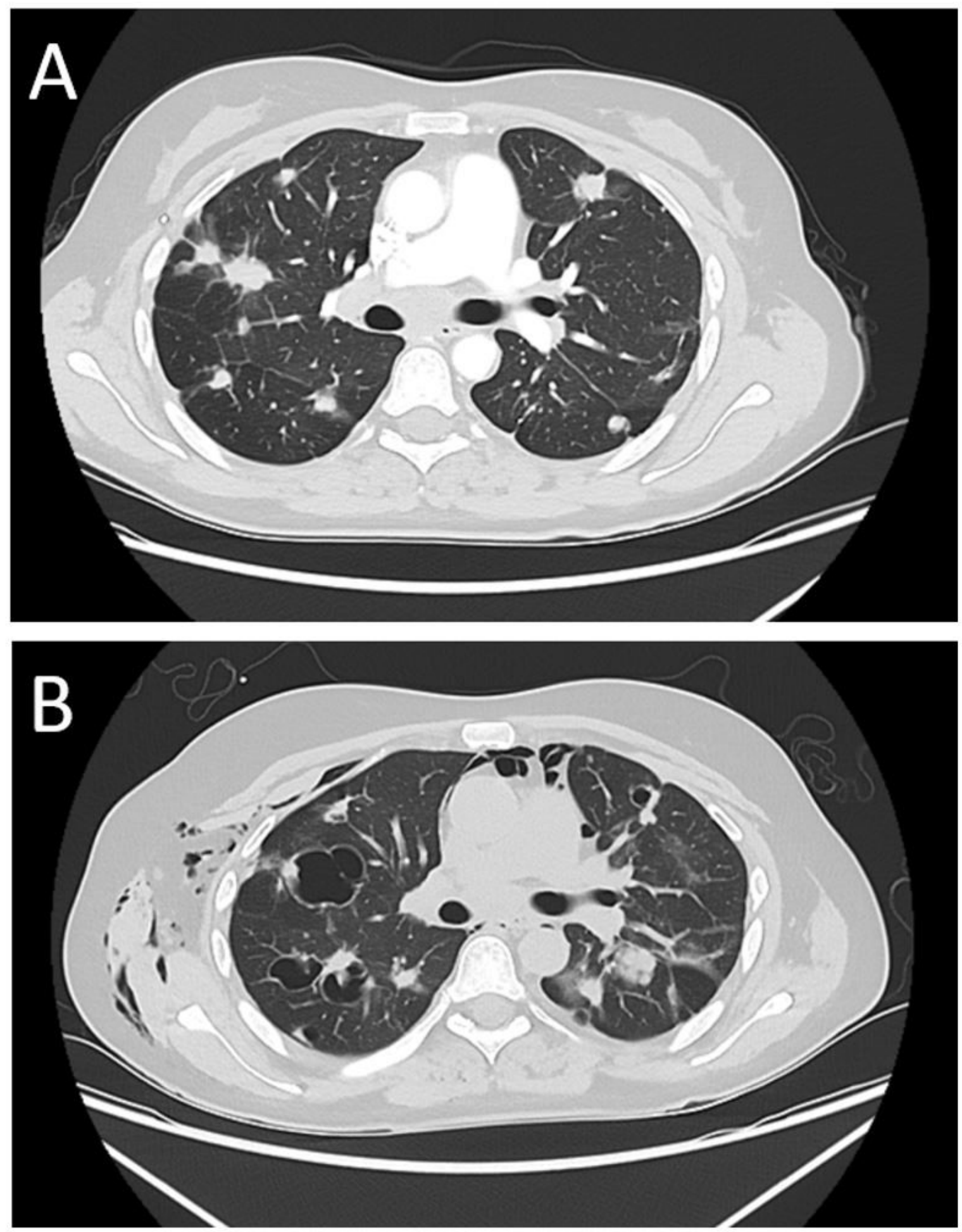

Figure 2

thoracic CT-scans before treatment with regorafenib (A) showing multiple bilateral nodular pulmonary metastases and 6 weeks after initiation of TKI-treatment (B) with bullous transformation and subcutaneous emphysema 